\title{
V-ATPase in cancer progression: Two sides of the same coin
}

\section{Mohd Saqib and Bibhuti B. Mishra}

News on: Hematopoietic stem cell specific V-ATPase controls breast cancer progression and metastasis via cytotoxic T cells by Sahoo et al. Oncotarget. 2018; 9:33215-33231. https://doi.org/10.18632/oncotarget.26061

Vacuolar ATPase (V-ATPase) is an ATP driven proton pump that is present on the membrane of intracellular vesicles like endosomes, lysosomes, where it is responsible for maintaining intracellular and vesicular $\mathrm{pH}$ under normal physiologic conditions. However, it is also expressed on the plasma membrane of cancer cells where it acidifies the extracellular environment, thus promoting growth and metastasis of cancer cells. The structure and function of V-ATPase are highly conserved across species. Structurally the V-ATPase has two domains: the membrane-bound " $\mathrm{V}_{0}$ " domain and the cytoplasmic " $V_{1}$ " domain [1]. Some of the V-ATPase subunits have multiple isoforms and their expression is tissue specific. For example, the "a" subunit that spans both $\mathrm{V}_{0}$ and $\mathrm{V}_{1}$ domains, has 4 isoforms and the "a2 isoform of V-ATPase" (a2V), is expressed on cells of the hematopoietic origin and endothelial cells [2]. Tumorderived a2 $\mathrm{V}$ plays a pathogenic role in the progression and metastasis of solid tumors. Tumor cells produce a2 V derived peptides that serve as potent chemo attractants for tumor associated neutrophils that subsequently produce growth factors and inflammatory eicosanoids to aid in metastasis of solid tumors [3, 4]. Although the role of tumor derived a2V as a tumor promoting factor is well established in various studies [5], [6] the role of immune cell-derived a2V in cancer progression has not been explored, given its expression by a number of innate and adaptive immune cells of hematopoietic origin.

In this work, Sahoo et al. examined the role of hematopoietic cell derived $\mathrm{a} 2 \mathrm{~V}$ in breast cancer growth and metastasis using a mouse model of breast tumor progression [7]. Previous studies have demonstrated that selective deletion of $\mathrm{a} 2 \mathrm{~V}$ in cancer cells blunts the cancer growth leading to the improved outcome. But, none of the published studies ever addressed the role of a2 $\mathrm{V}$ in immune cells that normally infiltrate the tumor microenvironment (TME), and affects the outcome of tumor progression. Sahoo et al. took the Cre-LoxP based approach of generating a conditional deletion of a2 $\mathrm{V}$ in hematopoietic stem cells (HSC) and demonstrated that a2 $\mathrm{V}$ in immune cells, unlike the cancer cells, possesses an anti-tumor function. a2 $\mathrm{V}$ conditional knock-out (KO) mice developed faster growing and more invasive breast cancer than the control littermates upon implantation of breast tumor in the mammary fat pad. This is a very interesting finding and opens many questions. To begin with, they analyzed the TME for the presence of infiltrating leukocytes by flow cytometry and cytokines/chemokines likely to contribute to the inflammation at the tumor site by targeted RNAseq. Interestingly, they discovered that the number of infiltrating $\mathrm{CD}^{+} \mathrm{T}$-helper (Th) and $\mathrm{CD}^{+}$ cytotoxic T cells (Tc) were significantly reduced, while the numbers of immune suppressive myeloid-derived suppressor cells (MDSC) were significantly increased in the TME of a2 $\mathrm{V} \mathrm{KO}$ mice. The TME of the a2V-KO mice is conducive for tumor growth by offering an antiinflammatory and anti-apoptotic milieu. In contrast, they attribute the smaller size of the tumor in control mice to a pro-inflammatory TME with a higher number of $\mathrm{CD}^{+} \mathrm{Th}$ and $\mathrm{CD}^{+} \mathrm{Tc}$ cells.

To decipher if the reduction of infiltrating $\mathrm{CD}^{+}$ Th and $\mathrm{CD}^{+} \mathrm{Tc}$ cells is due to a defect in the inability to migrate or a defect in their production, they analyzed various $\mathrm{T}$ cell subsets in the periphery (blood and spleen), site of maturation (thymus) and site of generation (bone marrow). They discovered that lack of a2V in HSCs causes a defect in production and maturation of both $\mathrm{CD}^{+}{ }^{+} \mathrm{Th}$ and $\mathrm{CD} 8^{+} \mathrm{Tc}$ cells in bone marrow and thymus, respectively. Therefore, the abundance of both the $\mathrm{CD}^{+}$ Th and $\mathrm{CD}^{+} \mathrm{Tc}$ cells is less in circulation and TME of a2V KO mice. Although the authors do not present any evidence of this defect mechanistically, they speculate that this defect in production and maturation of both $\mathrm{CD}^{+}{ }^{+} \mathrm{Th}$ and $\mathrm{CD}^{+} \mathrm{Tc}$ cells in a2V conditional knockout mice might be due (i) a defect in glycosylation and/ or (ii) cell death pathways. This speculation is based on previously published reports that deletion of a2 $\mathrm{V}$ relocates glycosyltransferase enzyme to endosomes and leads to enhanced cell death by inducing autophagy. Sahoo et al. went one step further to delineate which cells of the $\alpha \beta \mathrm{T}$ cell populations are important to control tumor growth and metastasis. For this, they depleted either the $\mathrm{CD}^{+}{ }^{+} \mathrm{Th}$ or the $\mathrm{CD}^{+}$Tc cells from normal mouse and monitored tumor growth and metastasis of implanted breast tumor cells. They demonstrate that lack of $\mathrm{CD}^{+} \mathrm{Tc}$ cells from the periphery results in larger, faster growing and metastatic breast tumor. The findings emerged from this study offers a new rationale for exploring the role of $\mathrm{a} 2 \mathrm{~V}$ in cancer biology and immunology in general. The work of Sahoo et al. raised a few important questions that deserve attention. The inherent lymphopenia observed in naïve a2 $\mathrm{V} \mathrm{KO}$ mice suggests that $\mathrm{a} 2 \mathrm{~V}$ might be playing a 
role in lymphopoiesis. Does a2V regulate T-cell numbers by controlling a crucial lineage commitment step of the hematopoietic progenitors, or does it affect T-cell survival by regulating cell death pathways? Does a2 V deficiency in HSCs compromise lymphoid cell generation at the expense of dysregulated myelopoiesis, as the metastasizing breast cancers are infiltrated with MDSC-like cells? Future experiments to address these questions may shed light on the mechanisms of antitumor effects of immune cellderived a $2 \mathrm{~V}$ especially in maintaining a T-cell pool in the tumor-bearing host.

Bibhuti B. Mishra: Albany Medical College, Albany, New York, USA

Correspondence to: Bibhuti B. Mishra,

email mishrab@amc.edu

Keywords: ATPase; tumor microenvironment; metastasis; cytotoxic T-cells; hematopoietic cells

Received: October 01, 2018

Published: October 16, 2018

\section{REFERENCES}

1. Cotter K, et al. Trends Biochem Sci. 2015; 40:611-22.

2. Marshansky V, et al. Biochim Biophys Acta. 2014; 1837:857-79.

3. Ibrahim SA, et al. Oncotarget. 2015; 6:33033-45. https://doi.org/10.18632/oncotarget.5439

4. Wculek SK, et al. Nature. 2015; 528:413-7.

5. Katara GK, et al. Mol Oncol. 2018; 12:208-23.

6. Kulshrestha A, et al. Mol Oncol. 2016; 10:789-805.

7. Sahoo M, et al. Oncotarget. 2018; 9:33215-31. https://doi.org/10.18632/oncotarget.26061

Copyright: Saqib. This is an open-access article distributed under the terms of the Creative Commons Attribution License 3.0 (CC BY 3.0), which permits unrestricted use, distribution, and reproduction in any medium, provided the original author and source are credited. 